\title{
Do Ponseti plasters delay gross motor milestones of South African children treated for idiopathic clubfeet?
}

\author{
M Street BSc, MBBCh, FCS(Orth)SA \\ Orthopaedic consultant \\ Y Ramguthy MBBCh, FCS(Orth)SA \\ Orthopaedic consultant \\ GB Firth MBBCh, FCS (Orth) SA, MMed (Orth) \\ Head: Paediatric Orthopaedics
}

Division of Orthopaedic Surgery, Chris Hani Baragwanath Hospital, University of the Witwatersrand, Johannesburg

\author{
Corresponding author: \\ Dr MR Street \\ Dept of Orthopaedics Wits Medical School \\ Tel: (011) 717-2538 \\ Fax: (011) 717-2551 \\ Email: mattstreet@vodamail.co.za
}

\begin{abstract}
Introduction: The Ponseti method for the treatment of idiopathic club foot is the gold standard of treatment in South Africa. A study in New York reviewed attainment of eight gross motor milestones in these children and found that independent ambulation was delayed on average by 2 months compared to unaffected children.

Methods: A retrospective review of gross motor milestones was performed in patients treated at a South African clubfoot clinic. All patients were ambulating independently at review and ages at attainment of three motor milestones were recorded (sitting, crawling and walking independently). The World Health Organisation (WHO) published the normal range for achievement of these milestones in six regions across the world. We compared the results of our patients to the 50th percentile in that study.

Results: Results show that patients in our study sat at a mean of 5.8 (standard deviation \pm 1.3 ) months and crawled at a mean of $8.3(\mathrm{SD} \pm 1.7)$ months, both equal to the WHO 50th centiles for unaffected children. Independent walking, however, was achieved at a mean of $15.3(\mathrm{SD} \pm 2.3)$ months which is statistically significantly different to the 50th centile for unaffected children in the WHO study $(\mathrm{p}<0.05)$. In our study, $86 \%$ of children achieved independent walking within 18 months. No statistical difference was found between unilateral and bilateral cases.

Conclusion: Children treated for idiopathic clubfoot (unilateral or bilateral) can expect no delays in sitting or crawling. Minimal delays in achieving independent walking of about three months can be expected with an $86 \%$ chance of walking within the accepted normal range for unaffected children.
\end{abstract}

Key words: Ponseti plasters, idiopathic club foot, motor milestones, children

http:/ / dx.doi.org/10.17159/2309-8309/2016/v15n2a8

\section{Introduction}

The Ponseti method ${ }^{1-3}$ of treating idiopathic clubfoot is the gold standard and the method used in this study at a tertiary level hospital clubfoot clinic.

Treatment of children with clubfoot involves serial weekly casting (above knee) with sequential manipulation of the clubfoot to achieve reduction of the navicular on the talar head with abduction of the foot to $60^{\circ}$. A percutaneous Achilles tenotomy is required in most cases to correct the remaining equinus deformity. Foot abduction orthoses (FAO) are worn full-time for three months and then at night and nap time up to the age of 4 years. ${ }^{1-3}$

On average 12 new cases of clubfoot per month are treated at our clinic. A number of parents expressed concern that the plasters and brace will hamper their child's future walking capability. Sala et al. from the New York Ponseti clubfoot centre showed that children treated using the Ponseti method showed pre-ambulatory gross motor milestone achievement delay of at most 1.5 months except for independent ambulation where a two-month delay was found. ${ }^{4}$ 
The purpose of this study was to assess whether there is a delay in patients with idiopathic clubfoot, treated with the Ponseti method in a South African setting, achieving three gross motor milestones as compared to the World Health Organisation (WHO) normal values as published in 2006. The 2006 growth reference study looked at the normal variation in ages of milestone achievement among healthy children. ${ }^{5}$ Children from five different countries were studied, namely Ghana, India, Norway, Oman and USA (Figure 1). ${ }^{5}$

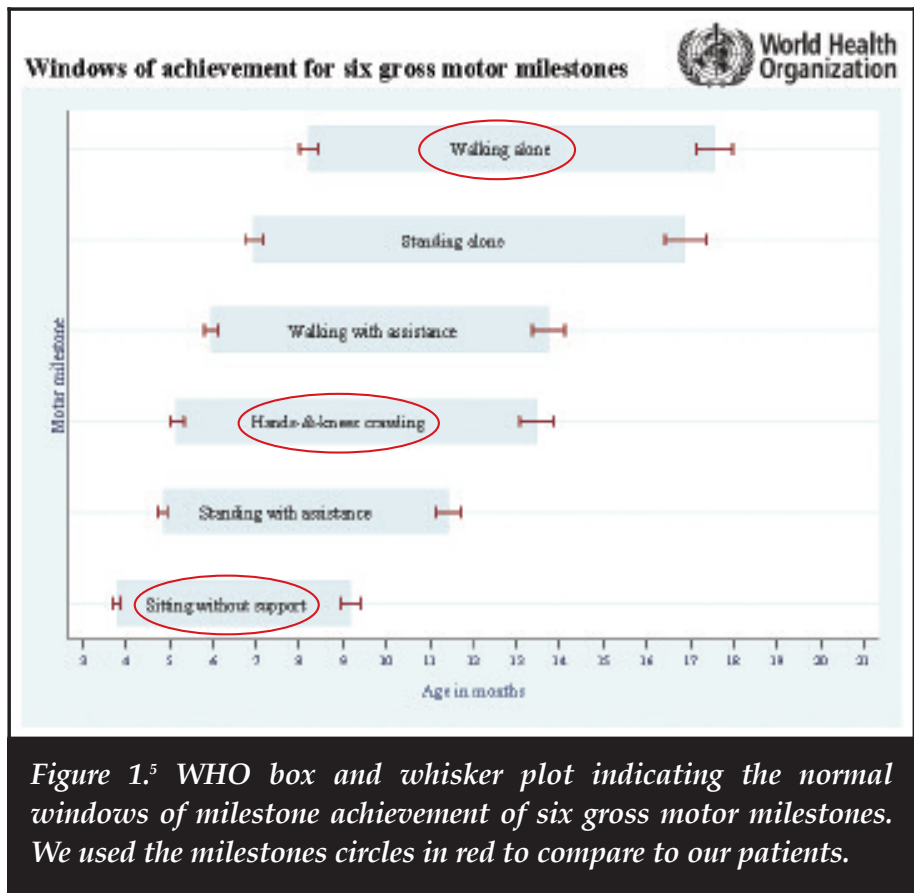

The advantage of using this reference data set was that it used five different countries, including two developing world countries, making it more reliable for our population.

\section{Aim}

The primary aim of the study was to assess the achievement of three gross motor milestones: independent sitting, crawling and independent walking. The achievement of these milestones was compared with age of achievement to the 50th percentile in the WHO study. A subsidiary aim was to compare delay in gross motor milestones in bilateral vs unilateral cases.

\section{Materials and methods}

This is a retrospective study of prospectively collected data from patients treated between 2008 and 2012 in a dedicated clubfoot clinic by the first author (MS).

Parents of children with clubfoot who had been treated by the Ponseti protocol who were ambulating independently were interviewed to determine the age of gross milestone achievement of independent sitting, crawling and independent walking. The age and gender of the patient, bilaterality and the age at commencement of plasters was noted.

Weekly plasters were applied until abduction of about 60 degrees was attained. The majority of patients required a tendo-Achilles tenotomy and placed into a cast for three weeks. Foot abduction braces (Steinbeek braces) were worn full-time for three months and then at night and nap time till 4 years of age.

All children with idiopathic clubfoot deformities presenting for follow-up between 1 January 2013 and 31 May 2013 were included. Exclusion criteria included teratological clubfeet (associated hip dysplasia, arthrogryposis, myelomeningocoele, etc.), any recurrence requiring recasting, and patients still in the casting phase, and patients in the first three months of full-time brace wear.

The WHO growth reference study ${ }^{5}$ used six parameters to assess achievement of gross motor milestones in children from five different countries. The data from this reference base was used to compare three things in our study population, namely time at which independent sitting, crawling and independent walking were achieved. Statistical analysis was done using STATISTICA (2009), using the one-sample Student t-test to compare the mean age of each milestone achievement and the WHO mean of unaffected children. A p-value $<0.05$ was considered statistically significant. The mean and range was calculated for the age at time of first cast application and number of casts used.

\section{Results}

Sixty-nine caregivers of children with idiopathic clubfoot deformities were interviewed. Forty-one (59\%) of the affected children were males and 28 were females (41\%). Forty-one $(59 \%)$ of the cases were bilateral and $28(41 \%)$ were unilateral.

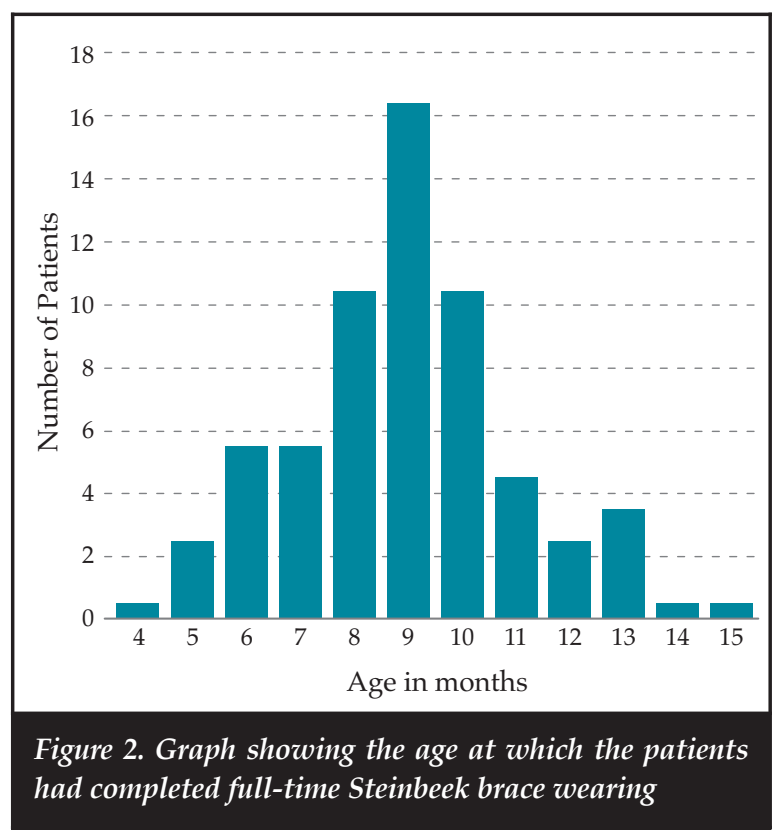


The mean number of plasters applied to achieve correction per patient was 10 (range 2-20) with the mean age at first plaster application seven weeks (range 5 days-35 weeks). Figure 2 shows the age at which the patients had completed their full-time FAO (Steinbeek brace) period of three months. Only nine out of the 69 patients were still in the abduction brace full time at 12 months of age.

Children in this study achieved independent sitting at a mean age of 5.8 months (SD 1.3 months). The WHO mean for sitting is 5.9 months on the 50th percentile for unaffected children. This result is not statistically significant $(\mathrm{p}=0.42$ ). The children in the current study achieved crawling at a mean age of 8.3 months (SD 1.7 months) which is the same as the 50th percentile in the WHO study; therefore, no statistical difference. Seven of the 69 children did not crawl prior to walking. Independent walking was achieved at a mean age of 15.3 months (SD 2.3 months) which is statistically significantly different compared to the WHO 50th percentile group which achieved independent walking at the age of 12 months $(\mathrm{p}<0.05)$ (Figure 3). Fifty per cent of children were independently walking by the age of 14 months. Eighty-six per cent were walking by the age of 18 months, which is the upper limit of the normal achievement of independent walking.

No statistically significant difference was found when comparing the children affected bilaterally to those affected unilaterally, with respect to independent sitting, crawling and independent walking.

\section{Discussion}

The population of 69 children in our group reflects similar characteristics to populations treated with the Ponseti method reported in the literature. In the current population $59 \%$ were male. There were slightly fewer males in this study group compared to the literature (range 60\%-75\% males) but bilateral cases (59\%) were equal to that described in the literature..$^{6-9}$

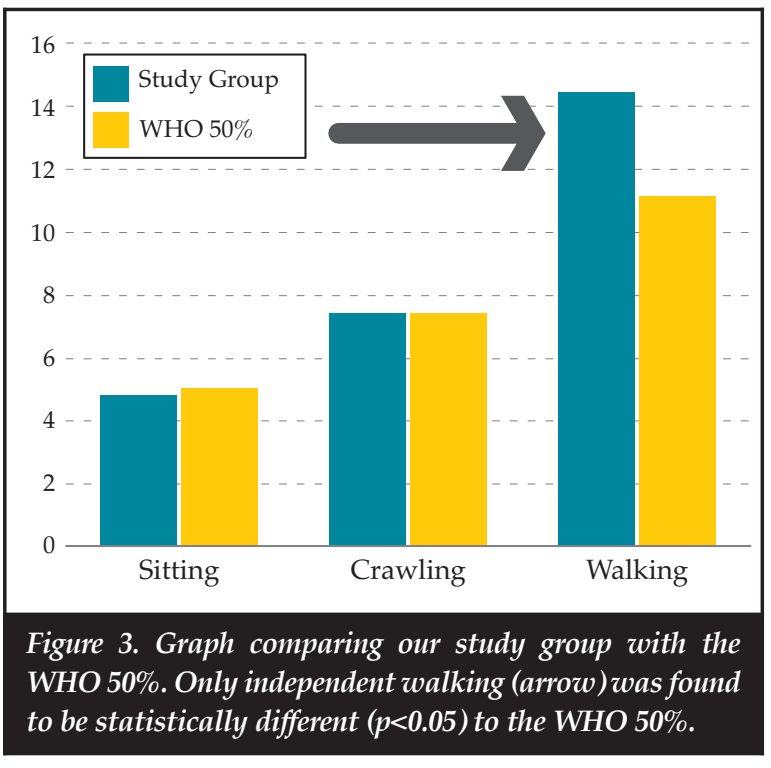

Ten plasters were on average required to obtain correction. This compares favourably to studies where plasters were started after six weeks ${ }^{10,11}$ but is more than other studies where plasters were started within the first few weeks of life. ${ }^{12,13}$

Sala et al. looked at achievement of gross motor milestones in 36 patients (53 feet). Children in their study had their first cast applied at a mean age of 15 days with a mean number of five casts (range 4-7). ${ }^{4}$ The current study is the first of its kind in an African setting. It had almost double the number of patients studied in New York ${ }^{4}$ with 69 children who had their first cast applied over a month later compared with this study (on average at 7 weeks with a range from 5 days to 35 weeks). There are many reasons for the later commencement of treatment in our study population. One reason is due to the referral patterns in our hospital system and the population's access to our facility. Many patients are referred from a clinic to a secondary facility and then on to our facility, which takes time. Other reasons include poor access to transport, poor education about the treatment modalities available for clubfeet and traditional beliefs. Despite the later application of the plasters in our study population the results of milestone achievement were similar to this study from New York. ${ }^{4}$ This makes the current findings more significant in an African context as the plasters were applied closer to the achievement of the early motor milestones and yet did not delay them more than when applied before the achievement of independent sitting.

The New York study looked at eight gross motor milestones and showed six of eight were delayed. Fifty per cent of the children in their study were ambulating independently by 14 months and $90 \%$ were ambulating independently by 18 months. ${ }^{4}$ They concluded that, although statistically significant, children receiving Ponseti plasters had minimal delays in achieving gross motor milestones with delays being at most two months. This, as in the current study, was not clinically significant. ${ }^{4}$

The current study has demonstrated similar results looking at three of these eight milestones. Fifty per cent were walking by 14 months and $86 \%$ ambulating independently by 18 months. Therefore $14 \%$ of the children fall outside the 99th percentile for independent walking as seen in the WHO study figures. Independent walking was delayed by a mean of three months compared to the 50th percentile of the WHO study figures. Although patients in this study received more plasters and were treated for longer, no additional delay of gross motor milestones were observed.

As a result parents can be reassured that although delays in walking are seen in these children, they are minimal, and not clinically significant. Children with clubfeet have an $86 \%$ chance of walking within what is considered a normal time frame (18 months). ${ }^{5}$ One can also inform them that sitting and crawling should not be affected.

The attainment of three gross motor milestones (independent sitting, crawling and independent walking) was recorded in the current study retrospectively. 
We chose to record only the above three milestones to ensure that parents could remember them easily and thus improve the reliability of our results. The literature supports this in a study done in Poland by MrożekBudzyn et al. where they found that parents were able to reliably recall the main gross motor milestones achieved when their children were 3 years of age. They supported the use of parent reporting of gross motor milestones retrospectively in children at low risk for developmental delays. ${ }^{14}$ This would be in keeping with our study population of children with idiopathic clubfeet. Another study done in Malawi used a developmental milestones checklist for parents of children aged 24 months or less. They found that the retrospective caregiver reports were both reliable and consistent on retesting, which supports the methodology of recalling milestones retrospectively by parents. ${ }^{15}$ Donoghue et al. concluded that a clinical interview assessing a child's milestones has limitations but its use is still justified. Walking age was particularly well recalled by the patients they interviewed. ${ }^{16}$ Another paper reinforcing that obtaining gross motor milestones retrospectively are reliable is Abubaker et al. who found that caregiver reports for milestones were reliable in a rural African setting. ${ }^{17}$

A limitation of our study was that it was retrospective and all children included had already started walking. Lastly we only interviewed the parents to record the milestones at one visit.

\section{Conclusion}

Children treated for idiopathic clubfoot can expect no delays in independent sitting or crawling when treated using the Ponseti protocol. Minimal delays in achieving independent walking (of about three months) can be expected but $86 \%$ of children will walk within the accepted normal range for unaffected children and this delay was not clinically significant.

\section{Compliance with Ethics Guidelines}

This article has been submitted to an ethical committee for approval. The content of this article is the sole work of the authors. M Street, Y Ramguthy and GB Firth have received no benefits of any form derived from any commercial party related directly or indirectly to the subject of this article.

\section{References}

1. Ponseti IV. Treatment of congenital club foot. J Bone Joint Surg Am. 1992;74:448-54.

2. Ponseti IV. Congenital clubfoot: fundamentals of treatment. 1st edition. Oxford; New York: Oxford University Press; 1996. p 140.

3. Ponseti IV. Clubfoot management. I Pediatr Orthop. 2000;20:699-700.
4. Sala DA, Chu A, Lehman WB, and van Bosse HJP. Achievement of gross motor milestones in children with idiopathic clubfoot treated with the Ponseti method. J Pediatr Orthop. 2013;33(1):55-58.

5. WHO Multicentre Growth Reference Study Group. WHO Motor Development Study: Windows of achievement for six gross motor development milestones. Acta Paediatrica Supplement. 2006;450:86-95.

6. Changulani M, Garg NK, Rajagopal TS, et al. Treatment of idiopathic club foot using the Ponseti method: initial experience. J Bone Joint Surg Br. 2006;88:1385-87.

7. Dobbs MB, Rudzki JR, Purcell DB, et al. Factors predictive of outcome after use of the Ponseti method for the treatment of idiopathic clubfeet. J Bone Joint Surg Am. 2004;86:22-27.

8. Chu A, Labar AS, Sala DA, et al. Clubfoot classification: correlation with Ponseti cast treatment. J Pediatr Orthop. 2010;30:695-99.

9. Herzenberg JE, Radler C, Bor N. Ponseti versus traditional methods of casting for idiopathic clubfoot. I Pediatr Orthop. 2002;22:517-21.

10. Cooke SJ, Balain B, Kerin CC, Kiely NT. Clubfoot. Current Orthopaedics. 2008;22:139-49.

11. Lourenço AF, Morcuende JA. Correction of neglected idiopathic club foot by the Ponseti method. J Bone Joint Surg [Br]. 2007;89:378-81.

12. Bor N, Coplan JA, Herzenberg JE. Ponseti treatment for idiopathic clubfoot. Minimum 5-year follow-up. Clin Orthop Relat Res. 2009;467:1263-70.

13. Gupta A, Singh S, Patel P et al. Evaluation of the utility of the Ponseti method of correction of clubfoot deformity in a developing nation. International Orthopaedics (SICOT). 2008;32:75-79.

14. Mrożek-Budzyn D, Kiełtyka MA, Majewska R. Validity and clinical utility of children development assessment using milestones reported by mothers. Przegl Epidemiol. 2014;68:71-75.

15. Gladstone M, Lancaster GA, Umar E, Nyirenda M, Kayira E, van den Broek NR, et al. The Malawi Developmental Assessment Tool (MDAT): the creation, validation, and reliability of a tool to assess child development in rural African settings. PLoS medicine. 2010;7(5):e1000273.

16. Donoghue EC, Shakespeare RA. The reliability of paediatric case-history milestones development. Med Child Neurol. 1967;9:64-69.

17. Abubakar A, Holding $\mathrm{P}$, Van de Vijver $\mathrm{F}$ et al. Developmental monitoring using caregiver reports in a resource-limited setting: the case of Kilifi, Kenya. Acta Pediatr. 2010;99(2):291-97.

This article is also available online on the SAOA website (www.saoa.org.za) and the SciELO website (www.scielo.org.za). Follow the directions on the Contents page of this journal to access it. 